\title{
DEUTSCHLAND-BILDER. IMAGOLOGISCHE UND KULTURGESCHICHTLICHE PERSPEKTIVEN
}

\begin{abstract}
Images of Germany. Perspectives from Imagology and Cultural History
Like all those pictures of other countries, which are treated in imagological research, images of Germany are also complex, mediated by social history and historical experience, misunderstanding, misrepresentation and ideological assumptions. On the whole, these images represent the expectations and experiences of the observer even as they can truly be connected with the history, or social and cultural affairs, of the population. Thus, these images appear to be reversible figures that are simultaneously true and the products of subjective perception.
\end{abstract}

KEY WORDS: Imagology, stereotype, nationalism, reflection, criticism, comparative (literature) studies, strangeness, modernity

Die Deutschen - ja wer sind sie? Wer sind, wenn wir, die Deutschen - bzw. diejenigen, die sich von „uns“ zu den Deutschen zählen möchten, „wir“? Und, wer sind sie, die Deutschen, wenn andere oder wir selbst von uns oder von anderen als „den“ Deutschen reden? Solche Fragen lassen sich aufwerfen und werden immer wieder aufgeworfen, stellen sich freilich auch deshalb immer wieder, weil sie sich nicht nur nicht abschließend, sondern stets nur bruchstückhaft, vorläufig und statt Eindeutigkeit immer auch Mehrdeutigkeit erzeugend, beantworten lassen. Wir befinden uns hier - um Elisabeth Beck-Gernsheim (Beck-Gernsheim, 1999) zu zitieren - im „Dschungel“ der Identitäten und ethnischer bzw. sozialer Kategorien. Ebenso bewegen wir uns auf einem Feld unterschiedlicher, divergierender und auch wechselnder persönlicher Zuschreibungen, von denen die eine Möglichkeit darauf zielt, sich gleichsam aus sich selbst heraus, aus der eigenen Lebensgeschichte, eigenen Vermögen und Erfahrungen, nicht zuletzt aus eigenen Zielsetzungen und Gruppenbezügen in individueller Freiheit zu bestimmen, während die andere Bezugslinie darin besteht, sich aus der Zugehörigkeit zu einer Gruppe, von der Familie über die Her- 
kunftsgruppe bis zur Nation, möglicherweise darüber hinaus als Europäer, Weltbürger, „Mensch“ und/oder Geschöpf Gottes zu bestimmen und hieraus Zughörigkeit und Selbstbewusstsein zu begründen. Auch dies ist unter den Bedingungen der Moderne, wie dies Ernest Renan bereits im Blick auf die nationale Zugehörigkeit beschrieben hat, keineswegs mehr schicksalhaft zugeteilt, sondern kann und muss vielmehr entscheidungsbedingt und situationsabhängig gesehen werden. Es bleibt Ernest Renans Formel von der Nation als einem ,plébiscite de tous les jours“ (Renan [1882], 1993, S. 309), freilich verbunden mit Erzählungen, auch Erfindungen großer Taten und großer Leiden, nicht zuletzt gegründet auf einem erheblichen Maß und einer entsprechenden Bereitschaft zum „Vergessen“: „Das Vergessen - ich möchte fast sagen: der historische Irrtum - spielt bei der Erschaffung einer Nation eine wesentliche Rolle, und daher ist der Fortschritt der historischen Studien oft eine Gefahr für die Nation." (ebd., S. 294) Gleichermaßen - im Anschluss an Renans ebenso emphatische wie prekäre Konstitution des nationalen Bewusstseins - gelten diese Bausteine und die mit ihnen verbundenen Aufgaben und Gefährdungen offensichtlich auch für die Konstitution einer nationalen (personalen) Identität. An der letztlich narrativen Herstellung (Marquard, 2000, S. 60) und Gestaltung individuell-personaler Identität, in deren Ensemble von Facetten dann das Nationale eine Tönung ausmachen kann, führt demnach kein Weg vorbei.

Immer aber, auf den Ebenen der Person ebenso wie auf der Ebene der Gruppenbezüge und auch darüber hinaus, geht es damit um Mehrdeutigkeiten, die im Hinblick auf Eindeutigkeit zur Auflösung anstehen und doch zugleich immer auch auf diesen Wegen zur Eindeutigkeit mit neuen Mehrdeutigkeiten konfrontiert sind, ja diese immer auch mit sich führen und aufs Neue erzeugen („Wann ist ein Mann ein Mann?"). ${ }^{1}$ Zugehörigkeit und Selbständigkeit, Beständigkeit und Wandel, Autonomie und Heteronomie heißen hier die Parameter, nach denen diese Prozesse des $\mathrm{Zu}-$ sich-selbst-Kommens und der Selbstverortung kartiert werden können, ohne dass sich dabei das Feld zugunsten irgendeiner Art von restloser Übereinstimmung auflösen ließe. Der Mensch, alleine auf sich selbst gestellt, ist ebenso wenig lebensfähig wie sich das völlige Aufgehen des Einzelnen in ein großes Ganzes als sinnvoll und menschengemäß vorstellen lässt. Beide Grenzpositionen sind wohl nur um den Preis des Verzichts bzw. der Vernichtung des Menschlichen (Besonderen) am Menschen zu haben. Hegel spricht hier mit Blick auf das Individuum zu Recht vom KonkretAllgemeinen (Hegel [1821], 1970, S. 54). Er sieht wie viele andere vor und nach ihm den Menschen damit vor allem als eine Schnittstelle von jeweils Allgemeinem und Besonderem an, in dessen Form und Funktion sich eben Widersprüche jedweder Art treffen und ihre jeweils konkrete, nie ganz generalisierbare, zeitweilige Gestalt oder Vermittlung finden (müssen). In ihrer konkreten Individualität erscheinen sie

\footnotetext{
${ }^{1}$ Judith Butler spricht hier zu Recht von „Gender Trouble“ (Butler, 1991).
} 
jeweils aber so gemischt, dass keiner ganz dem anderen gleicht, es sei denn eben in dieser Eigenschaft, jeweils allgemeine Setzungen in einer besonderen Weise und auf einer nur in diesem Einzelfall realisierten Weise darzustellen.

\section{Gruppenprozesse und Identität(en)}

„So wie alle, so wie kein anderer", nach diesem Grundsatz lassen sich nicht nur menschliche Gesichter beschreiben, sondern eben auch die Ansprüche, die darauf zielen, „ein“ Mensch zu sein. Der alte Satz „individuum est ineffabile“ (= Aristoteles, 1995) lässt sich in diesem Sinne nicht nur als Begrenzung von Aussagen lesen, die wie „wissenschaftliche“ auf ein Allgemeines zielen und deshalb am Individuum in seiner jeweils zufälligen Besonderheit ihre Grenze erfahren. Der Satz beschreibt vielmehr auch die Würde und Unhintergehbarkeit des Individuums (Frank, 1986), das sich eben nicht darauf beschränken bzw. daraus begründen lässt, Repräsentant einer (wie auch immer definierten) Gruppe zu sein. Es gibt, so müsste also in diesem Sinne die paradoxe Antwort auf die eingangs gestellte Frage lauten, so viele Deutsche wie es Menschen auf der Welt gibt, und wenn sie denn alle „Deutsche“ sein können, so könnten sie ebenso sehr auch als Chinesen gesehen werden usw. Entsprechend hat der englische Romancier und Literaturkritiker Julian Barnes einen Satz des französischen Schriftstellers Gustave Flaubert (1821-1880) kommentiert: „,Ich bin im selben Maße Chinese wie Franzose', erklärte Flaubert. Das heißt auch nicht mehr Chinese: wäre er in Peking geboren worden, hätte er die dortigen Patrioten zweifellos auch enttäuscht" (Barnes, 1989, S. 191). Bereits vierhundert Jahre vor Barnes hatte der französische Schriftsteller Michel de Montaigne um das Jahr 1580 etwas Ähnliches notiert:

Non parce que Socrate l'a dit, mais parce qu'en vérité c'est mon humeur, $\ldots \mathrm{j}^{\mathrm{j}}$ estime tous les hommes nos compatriotes et embrasse un Polonais comme un Français, postposant cette liaison nationale à l'universelle et commune (...) Nature nous a mis au monde libres et déliés. (Montaigne, 1972, S. 235)

Diesen Befunden, die noch wesentlich zu erweitern und selbstverständlich auch fundierter darzustellen wären, scheinen nun aber auch zumindest zwei Beobachtungen bzw. Erfahrungen zum Umgang mit Großgruppen-Zugehörigkeiten entgegen zu stehen: Zum einen ist es uns - und auch hier können wir den Zeugnissen nach an die Anfänge der Kulturgeschichten der Menschheit zurückgehen offensichtlich sehr vertraut, in Gruppenbezügen zu denken. Schnell sind wir dabei, Menschen ihrem Äußeren, zumal ihren Sprachen und Bekenntnissen nach in Gruppen zu ordnen, wobei diese Ordnungen häufig ziemlich schematisch ausfallen, nach dem Muster: Was bei uns „so“ ist, muss bei ihnen „anders“ sein, häufig wird sogar nach dem Muster von Herodots Bericht über die Ägypter - genau das direkte Gegen- 
teil angenommen. „Wie die Ägypter“, so schreibt Herodot im zweiten Buch seiner Historien,

einen Himmel haben, der besonders ist, und einen Fluß, der eine andere Art hat als die übrigen Flüsse, so haben sie sich auch in fast allen Stücken Gewohnheiten und Sitten zugelegt, die denen anderer Menschen grad entgegengesetzt sind. So gehen bei ihnen die Frauen auf den Markt und handeln, die Männer aber sitzen zuhaus und weben. (...) Die Frauen lassen Wasser im Stehen und die Männer im Hocken. Ihre Notdurft verrichten sie in den Häusern, Essen aber tun sie draußen auf den Straßen und sagen dazu, was unanständig, aber notwendig ist, solle man im Verborgenen tun, was aber nicht unanständig ist, vor aller Augen. (Herodot, 1991, S. 139)

Das Unvertraute bei anderen, so hat es der amerikanisch-österreichische Germanist Egon Schwarz (1976) bereits vor Jahren in einem auch heute noch lesenswerten Essay zum Gruppendenken in Leben und Literatur (Untertitel zu Die sechste Schwierigkeit beim Schreiben der Wahrheit) dargestellt, wird dann zumeist als das Typische und, da es als typisch ,anders“ gilt, in einem zweiten Schritt dann auch gleich als das „Schlechtere“ im Vergleich zum Eigenen angesehen. In dieser Weise hatte Herodot bereits die Ägypter geschildert, in dem er deren Lebensweise als diametral entgegengesetzt zu den Mustern darstellte, die den Griechen, seinem Publikum, vertraut waren.

Freilich ist es mit Zuschreibungen von Individuen zu Großgruppenverbänden so eine Sache, wie jeder weiß, der sich schon einmal mit entsprechenden Zuordnungsversuchen befasst hat: So nahe sie liegen und zu entsprechenden Annahmen oder Aussagen verführen, so schwierig ist es im Einzelfall, einen Deutschen von einem Schweizer, einen kenianischen Hawaiianer von einem äthiopischen Kanadier ${ }^{2}$, einen Vietnamesen aus der ehemaligen DDR von einem Kambodschaner aus Frankreich usw. zu unterscheiden. Dass es sich bei solchen Entscheidungen und den sie begründenden Erfahrungen häufig weniger um „Fakten“ handelt, die von den „Sachen“, also den Eigenschaften der zu Beobachtenden ausgehen, als vielmehr um einen Mangel an Erfahrung und Vertrautheit der Beobachter, hatte der bedeutende Soziologe Georg Simmel (1858-1918) bereits im Rahmen seiner Studien zur sozialen Differenzierung in den letzten Jahrzehnten des 19. Jahrhunderts erkannt:

Wir vernehmen von Reisenden, und können es in gewissem Maße auch selbst beobachten, dass bei der ersten Bekanntschaft mit einem fremden Volksstamme alle Individuen desselben ununterscheidbar ähnlich erscheinen, und zwar in um so höherem Maße, je verschiedener von uns dieser Stamm ist; bei Negern, Chinesen u. A. nimmt diese Differenz das Bewusstsein so sehr gefangen, dass die individuellen Verschiedenheiten unter jenen völlig davor verschwinden. Mehr und Mehr aber treten sie hervor, je länger man diese zunächst gleichförmig erscheinenden Menschen kennt; und entsprechend verschwindet das stete Bewusstsein des generellen und fundamentalen Unterschiedes zwischen uns und ihnen; (...) die Beobachtung zeigt, dass sie in demselben Maße als uns

\footnotetext{
${ }^{2}$ Vgl. dazu die wunderbaren Ausführungen von Doug Saunders über hawaiianische Kanadier und kanadische Australier (Saunders, 3. November 2015).
} 
homogener erscheinen, in dem sie als unter sich heterogener erkannt werden: die allgemeine Gleichheit, die sie mit uns verbindet, wächst in dem Verhältnis, in dem die Individualität unter ihnen erkannt wird. (Simmel [1888], 1989, S. 26; Hervorhebung im Text)

Natürlich soll es hier nicht darum gehen, eine weltweit vertraute, wohl mit den anthropologisch beschreibbaren Orientierungsbedürfnissen von Menschen verbundene Neigung zur Zurechnung von Individuen zu Gruppen einfach in Abrede zu stellen oder ebenso einfach überwinden zu wollen; wohl ist aber im Anschluss an Simmel auf die Notwendigkeit der Reflexion des eigenen Standortes, der eigenen Zurechnungen und der damit verbundenen Grenzen hinzuweisen; eine Position, die sich auch auf die andere Weise beziehen lässt, in der Menschen offensichtlich dazu neigen, Großgruppenbezüge herzustellen und auszuführen. Es geht dabei um die Neigung, das Bedürfnis, den Hang von Individuen, „irgendwo“ dazu gehören zu wollen, eine Intention nach Gruppenbezug, die sich wohl ebenfalls anthropologisch, soziologisch und psychologisch beschreiben lässt und die doch auch - zumindest in den polyvalenten Orientierungsmustern der Moderne $^{3}$ - gerade dahingehend reflektiert werden muss, dass es für Menschen nicht immer nur eine, sondern in der Regel mehrere/viele Zugehörigkeiten gibt. Es handelt sich dabei um eine Beobachtung, die sich in der modernen Rollensoziologie ebenso wiederfindet wie in entsprechenden Theorien sozialer Differenzierung (von Simmel bis Luhmann), und die aktuell u. a. in den Konzepten „,multipler Identitäten“(Keupp, 1999) zum Ausdruck kommt.

Auch geht es hier nicht darum, dieses Bedürfnis und die entsprechenden Handlungen zur Bildung von Gruppen und Gemeinschaften per se in Frage zu stellen oder gar zu delegitimieren. Gleichwohl ist darauf hinzuweisen, dass es viele Möglichkeiten der Loyalitäten und damit auch der Sicherung und Weiterführung von Identitäten und Lebensmustern gibt. Auch wenn manche von diesen, so die Zuordnung zu sprachlich-kulturell oder politisch definierten Großgruppenverbänden wie Nationen oder religiösen Denominationen, zeitweise historisch im Vordergrund standen oder stehen und damit verbunden eine entsprechende Deutungskraft und Kohäsion von ihnen auszugehen vermag. ,Wo immer jemand auf eine Rolle festgelegt, sozusagen festgenagelt wird“, so der Politologe Christian Graf von Krockow in seiner Studie zur ,politischen Kultur des Konflikts“" von 1983,

die in alle anderen Rollen durchschlägt und die als ein Schicksal, als gleichsam naturhafte über den Rollenträger verfügt wird - der Deutsche, türkische Gastarbeiter, Neger, Jude, Homosexuelle, Zigeuner, die Frau, der Radikale, Kapitalist, Kommunist, was immer - : da ist das in der modernen Gesellschaft ein Signal der Intoleranz, der verweigerten Freiheit. Verfemung ist dann mindestens heimlich immer schon vorhanden, und die Verfolgung duckt sich zum Sprung. (von Krockow, 1983, S. 67; Hervorhebung im Text)

\footnotetext{
${ }^{3}$ Anthony Giddens hat diese Aspekte im Blick auf die Erfahrungen eines Lebens unter den Bedingungen der Moderne in die beiden Prozesse des Dis-Embedding (Entbettung) und Re-Embedding (Rückbettung) gefasst (Giddens, 1995, S. 28-74).
} 
Dies ist freilich nicht nur eine Frage der Moral bzw. einer, so wie von Krockow es sich in dieser Studie vorgenommen hat, gelebten „politischen Kultur“, sondern in gewissem Sinn Voraussetzung und zugleich Folge der mit der Moderne verbundenen Differenzierungs- und Individualisierungsprozesse selbst. Da, wo unter den Bedingungen einer „reflexiven“ Moderne (Beck, Giddens \& Lash, 1996) von Großgruppenzuordnungen gesprochen wird, können diese immer nur im Modus des „als ob" in einem gleichsam historischen oder mythisch verbrämten, also ideologisch hergestellten bzw. bestimmten Rahmen angeführt und ausgearbeitet werden. Sicherlich ist dies ein Grund mehr, um national ausgerichtete Großgruppenbilder eben in diesem Sinne als Machwerke/Konstruktionen aufzufassen und diese entsprechend analytisch-reflexiv zu erschließen. ${ }^{4}$

Ziel dieser vergleichsweise langen Einleitung zum Thema der Deutschlandbilder war es, zunächst einmal den Stellenwert und die Machart von Völkerbildern als Hilfskonstruktionen im Hinblick auf die Orientierungsbedürfnisse von Menschen einerseits sowie als Mittel der Zurechnung, der Zuschreibung und auch der Herstellung von Großgruppenverbänden - also auch als Mittel der künstlichen, ideologischen Herstellung von Gemeinschaften - andererseits vorzustellen. Damit sollte eine Ebene angesprochen werden, auf der sich „Deutschland-Bilder“ beschreiben, untersuchen und entsprechend reflektieren lassen, um sie im Sinne dieser letztgenannten Tätigkeit dann auch mit Blick auf weitergehende und aktuelle Rahmenbedingungen, Entwicklungen, Lücken, Widersprüche und wechselseitige Spiegelungen zu betrachten. Im Folgenden werde ich das Thema in weiteren vier Schritten behandeln: Zunächst werde ich im zweiten Abschnitt einige Deutschlandbilder: Wer sind die Deutschen? vorstellen, um von hier aus bereits die Ambivalenz, die Verschiedenheit der Facetten und Rahmensetzungen anzusprechen. Dazu gehört auch der Umstand, dass es sich bei gruppenspezifischen Bildern häufig um Wechselspiegelungen und Kippfiguren handelt, sich also die Beobachter selbst in den von ihnen wahrgenommenen und übermittelten Bildern wiederfinden lassen. Schon hier wird die Differenz von Auto-Images/images und Hetero-Images/mirages anzusprechen sein, die dann im dritten Abschnitt zum Themenfeld der Images und der Imagologie als einem der zentralen Arbeitsfelder der Komparatistik (Corbineau-Hoffmann, 2000, S. 171-185) weiterführt. Schließlich werde ich im vierten Abschnitt eine Übersicht über die Geschichte und die unterschiedlichen Ausprägungen von Deutschlandbildern geben und abschließend im fünften Abschnitt einige Punkte ansprechen, die sich auf eine weitergehende kultur- und literaturwissenschaftliche, komparatistische Arbeit und entsprechende Perspektiven beziehen.

\footnotetext{
${ }^{4}$ Außerordentlich instruktive Beispiele für den hergestellten Charakter nationaler Geschichten und ihrer Stoffe und Helden finden sich unter dem unscheinbar daherkommenden Titel Lebendige Vergangenheit gesammelt (Graus, 1975).
} 


\section{Die Deutschen - wer sind sie?}

In der Einleitung zu einem der in der zweiten Hälfte des 18. Jahrhunderts am meisten diskutierten und verbreiteten Bücher, der 1780 erstmals erschienenen und wegen ihrer kolonialkritischen Schärfe auch sogleich verbotenen Histoire des deux Indes, die in Zusammenarbeit mit dem Aufklärer und Enzyklopädisten Denis Diderot (1713-1784) entstand, gibt ihr Verfasser Abbé Raynal (1713-1796) zunächst eine Übersicht der zivilisatorischen und politischen Entwicklungen Europas und kommt dabei auch auf die Rolle der einzelnen Länder und Herrschaften Europas zu Beginn der frühen Neuzeit zu sprechen:

Das durch die Streitigkeiten der Kaiser und Päpste und durch innerliche Kriege lange Zeit zerrüttete Teutschland hatte unlängst eine ruhigere Lage gewonnen. Der Anarchie war Ordnung gefolgt, und die Völker dieses großen Lands ohne Reichtümer, ohne Handel, aber Krieger und Feldbauleute, hatten von ihren Nachbarn nichts zu befürchten und konnten ihnen nicht furchtbar sein. Die LehnsRegierungsform war dort dem menschlichen Geschlecht minder erschrecklich als in andern Ländern. Überhaupt regierten die verschiedenen Fürsten dieses weitläufigsten Teils von Europa ihre Länder ziemlich gut. Sie missbrauchten ihre Gewalt wenig, und wenn der ruhige Besitz seines Erbteils dem Menschen die Freiheit ersetzen kann, so waren Teutschlands Bewohner glücklich. (Raynal \& Diderot, 1988, S. 28)

Eine solche Einschätzung nimmt sich zunächst einmal verwunderlich aus, sind wir es doch eher gewohnt, auch in den älteren Verhältnissen Deutschlands Zurückgebliebenheit, Despotismus, Militarismus (Elias, 1989, S. 23; Craig, 1982, S. 267-272) und Gängelei am Werke zu sehen, ein Unbehagen und eine Form der Selbstdisziplinierung und Selbstunterdrückung vorzufinden, die sich dann als Grundlage und Motivkomplex für aggressive politische Programme und deren rücksichtslose Umsetzung benennen lassen. Neben anderen bemerkenswert ist in diesem Zusammenhang immer noch die berüchtigte „Hunnenrede“ Kaiser Wilhelms II. am 27. Juli 1900 bei der Verabschiedung eines deutschen Truppenkontingents zu einer gemeinsamen europäischen Aktion, die den antikolonialistisch gerichteten Boxeraufstand in China niederschlagen sollte.

Bewahrt die alte preußische Tüchtigkeit, zeigt euch als Christen im freudigen Ertragen eurer Leiden, (...) gebt an Manneszucht und Disziplin aller Welt ein Beispiel. Ihr wißt es wohl, ihr sollt fechten gegen einen verschlagenen, tapferen, gut bewaffneten, grausamen Feind. Kommt ihr an ihn, so wißt: Pardon wird nicht gegeben, Gefangene werden nicht gemacht. Wer euch in die Hände fällt, sei in Eurer Hand. Wie vor tausend Jahren die Hunnen unter ihrem König Etzel sich einen Namen gemacht, der sie noch jetzt in der Überlieferung gewaltig erscheinen läßt, so möge der Name Deutschlands in China in einer solchen Weise bestätigt werden, daß niemals wieder ein Chinese es wagt, etwa einen Deutschen auch nur scheel anzusehen. (Ostasieninstitut Ludwigshafen, o. J., URL; vgl. für eine im Nachhinein gemäßigtere Fassung: Pross, 1983, S. 44f.)

Freilich war dieser Ton Kaiser Wilhelms durchaus geeignet, nicht nur die Chinesen zu erschrecken, sondern auch die Nachbarn der Deutschen zu alarmieren. 
Entsprechend wurde dieser Text nach dem völkerrechtswidrigen Überfall auf Belgien zu Beginn des Ersten Weltkriegs und im Zusammenhang des nachfolgenden Gas- und Propaganda-Krieges immer wieder aufgenommen. Zudem diente er vor allem in der ersten Hälfte des 20. Jahrhunderts auch zur Charakterisierung der Deutschen überhaupt, die sich in den Kriegs- und Völkerrechtsverbrechen des Zweiten Weltkriegs, zumal in der alle zivilisatorischen Standards und Vorstellungen von Menschlichkeit untergrabenden Vernichtung der europäischen Juden während der NS-Herrschaft, in einer Weise gezeigt haben, die bis heute unfassbar und unvergleichlich geblieben ist.

Dem gegenüber ist die ältere Wahrnehmung der Deutschen keineswegs auf diese Linie festzulegen, sondern verbindet Elemente der Rückständigkeit mit solchen der Wertschätzung und der Offenheit, wobei die Rückständigkeit gerade auch als Hinweis auf mangelnde Bedrohlichkeit gesehen werden konnte. Während sich heutige Deutsche immer auch noch als besonders ,,arbeitsam“ sehen und allenfalls in den letzten Jahrzehnten in dieser übergroßen Wertschätzung der Arbeit eine Art Selbstgefährdung erkennen wollen (Greiffenhagen, 1983, S. 404; dazu auch Bausinger, 2000, S. 26-31), stellen Arbeit und Disziplin in den Augen Raynals und Diderots eher eine Ausnahme dar:

Nur in den freien und in den großen Hansebund getretenen Städten fand sich Handlung und Arbeitsamkeit. (...) Das Geld war rar, der Landmann verkaufte einige Pferde ... Die Fürsten verhandelten noch keine Menschen. Die Tafel und eine Menge Pferde war die einzige übliche Pracht. Die Vornehmen und die Geistlichkeit betranken sich, ohne Unruhen im Staat zu stiften. (...) Die Sitten waren wild und noch in den zwei nachfolgenden Jahrhunderten waren die teutschen Truppen mehr durch ihre Grausamkeit als durch ihre Kriegszucht und ihren Mut bekannt. (Raynal \& Diderot [1780], 1988, S. 28)

Deutschland erscheint hier als ein Land von Menschen in rückständiger Lage, mit Seltsamkeiten und mangelnder Selbststeuerung behaftet, zugleich aber nicht als ein Land, von dem größere Bedrohungen ausgehen konnten.

Nicht viel anders erschienen Deutschland und die Deutschen noch knapp hundert Jahre später einem amerikanischen Besucher, der nach Berlin zum Studium gekommen war und erst einmal ein Gymnasium besuchte, um sich hier mit der deutschen Sprache vertraut zu machen. Der US-Amerikaner Henry Adams (1838-1918), Abkömmling einer der großen Elitefamilien Bostons und späterer Geschichtsprofessor, veröffentlichte im Jahr 1904 seine Autobiographie The Education of Henry Adams, ein Werk, das noch heute - wohl ganz zu Recht - als eines der Standardwerke nicht nur des literarischen Kanons der USA gesehen wird, sondern auch eine transatlantische Kulturgeschichte des 19. Jahrhunderts umfasst. In diesem auch heute noch lesenswerten Buch berichtet er auch von seinem, durchaus durch die Begeisterung für die deutschen Philosophen und Schriftsteller um und nach 1800 angeregten, Berlin-Aufenthalt Ende der 1850er Jahre: 
Im Jahre 1858 war Berlin eine arme, aufgeweckte Stadt, einfach, schmutzig, unzivilisiert und in den meisten Hinsichten abscheulich. Das Leben dort war primitiver, als ein amerikanischer Junge sich hätte vorstellen können. Überwältigt von militärischen Methoden und bürokratischer Kleinlichkeit, begann Preußen sich eben erst von den Fesseln im Innern zu befreien. Von Disziplin abgesehen, gab es kaum irgendwelche öffentliche Tätigkeit. (...) Die deutschen Sitten, selbst bei Hofe, waren manchmal roh, und die deutsche Gründlichkeit war gewöhnlich nur Routine. (Adams, 1953, S. 126f.)

Furcht empfindet Adams freilich keine, allerdings ist er entsetzt, z. T. voller Mitgefühl, aber auch Verachtung für das deutsche Schul- und Bildungssystem:

Die willkürliche Übung des Gedächtnisses war bestürzend; die Anspannung, die das Gedächtnis zu erdulden hatte, war eine Art Folter, und die Taten, die die Knaben ohne Klage vollbrachten, waren erbarmungswürdig. Keine andere Fähigkeit als das Gedächtnis schien anerkannt zu werden. Am wenigsten macht man von der Vernunft Gebrauch, der analytischen, synthetischen oder dogmatischen. Die deutsche Regierung ermutigte das selbständige Denken nicht. (Ebd., S. 128)

Und Adams findet auf den nachfolgenden Seiten noch abschätzigere Worte für das deutsche Bildungs- und Erziehungssystem: „Die deutsche Universität hatte Adams ein Fehlschlag geschienen, aber die deutsche höhere Schule war fast etwas wie ein sträflicher Unfug" (ebd., S. 130). Er findet aber dann doch auch Zeit für Biergärten, die Freude an Heinrich Heine, unter dessen Eindruck er überhaupt beschlossen hatte, nach Europa bzw. nach Deutschland zu gehen, die Musik deutscher Komponisten, namentlich Beethovens, und nicht zuletzt die schönen Landschaften, die ihm rückblickend als eine Gegenwelt zu Kultur und Bildung in Berlin erscheinen: „Dennoch sang sein Herz wie ein Vogel, als endlich der April herankam, und irgendein guter Geist eine Wanderung durch Thüringen vorschlug“ (ebd., S. 133). Gegenüber dem Vater freilich blieb er dabei, „dass Berlin die beste aller Erziehungen im besten aller Deutschlande sei“ (ebd.). Die Ambivalenz seines DeutschlandBildes fasst er selbst zusammen, wenn er schreibt: „Obgleich er darauf bestand, dass sein Glaube an die deutsche Philosophie und Dichtung erhaben war, gelang es ihm nicht, sich dem deutschen Denken zu nähern, und er vergoß nie eine Träne der Rührung über den Werken Goethes und Schillers" (ebd.).

Eine Unbestimmtheit der Mitte, die sich ebenso sehr in Formlosigkeit und Grobianismus wie in übersteigerter Formstrenge - der Ablehnung zivilisierter Umgangsformen ebenso wie der Hochschätzung und Überbewertung der Disziplin zeigt, scheint in diesem Sinne charakteristisch zu sein für die Menschen, die in jenen Landschaften in der Mitte Europas über längere Zeit gelebt haben, die sich bis weit ins 18. Jahrhundert hinein unter dem Sammelbegriff der „deutschen lande“, später dann unter dem Stichwort „Deutschland - aber wo liegt es“ (Friedrich Schiller), schließlich als zweites und drittes Deutsches Reich zusammenzufassen suchten. Das erste deutsche Reich war schon insoweit eine irritierende, wenn nicht gar in die Irre führende Bezeichnung gewesen, als dieses 911 oder 919 u. Z. gegründete Reich 
tatsächlich mehr als zwei Drittel der Zeit seines Bestehens lediglich die Bezeichnung „Imperium Romanum“ führte. Der Zusatz „nationes tedescae“, also immer noch nicht „deutsches Reich“, wurde erst 1512 angehängt und firmierte dann bis zur Auflösung des Reiches 1806. Dass der Begriff Nation zu dieser frühen und auch noch in späterer Zeit nicht der moderne, auf Homogenität der Bevölkerung und gemeinsamem politischen Willen aufruhende Nationen-Begriff des 19. Jahrhunderts und des entsprechend in dieser Zeit sich ausformenden Völkerrechts war, sondern ein vielfach ständisch, ethnisch, kulturell gebrochenes, ,multikulturell“ zusammengesetztes Gebilde ansprach, muss natürlich ebenfalls berücksichtigt werden. „Der Begriff ,Nation' bezeichnete dagegen im Mittelalter die Großstände und wandelte sich dann zu einem ständischen Verfassungsbegriff: Die ,deutsche Nation' waren die auf dem Reichstag zu Regensburg versammelten Reichsstände, also Adel und Geistlichkeit; das einfache Volk gehörte nicht dazu" (Schulze, 1989, S. 27). Und dass diese Vorstellung eines gemeinsamen politischen, sozialen und kulturellen Verbundes auch noch im 19. Jahrhundert durchaus nicht überall auf der Ebene der einfachen Leute, also des Volkes angekommen war, lässt sich u. a. einer satirischen Darstellung entnehmen, in der der Dramatiker Christian Friedrich Grabbe (1801-1836) in seinem 1835/36 geschriebenen historischen Drama Die Hermannsschlacht eben jenen Cherusker Hermann ${ }^{5}$ bei dem Versuch zeigt (und zunächst scheitern lässt), den Menschen in den „deutschen Landen“ so etwas wie Nationalbewusstsein zu vermitteln:

\footnotetext{
Hermann: Deutschland!

Einige in seinem Heer: Er spricht oft davon. Wo liegt das Deutschland eigentlich?

Einer: Bei Eger, wie ich glaube, oder irgendwo im kölnischen Sauerlande.

Zweiter: Ach was! Es ist chattisches Gebiet.“

Erst als Hermann sich darauf besinnt: „Ich muss mit geringeren aber näheren Mitteln wirken“ und den Bauern Grüttemeier an den Verlust seiner Ochsen durch die Römer erinnert: „Ein Manipel stürmte in dein Haus, schlachtete, briet und fraß sie und gab dir nichts ab!“ entsteht so etwas wie ein Kollektivbewusstsein und anschließend doch noch die gewünschte Kampfbereitschaft. Mit dem fürchterlichen Ausruf „Ha!“ stürzen sich die endlich die „Die Deutschen“ genannten westfälischen Bauern auf die Römer. (Grabbe zitiert nach Schulze, 1989, S. 29f.)
}

Unsicherheit und Orientierungslosigkeit als Erfahrungen bei einem kulturell, das heißt durch religiöse wie durch politische und literarisch-pädagogisch Erwartungen gesteigerten Bedürfnis', ja Druck, nach Festigkeit und Form als Grundzüge eines aus den Fremd- und Eigenbeobachtungen sich konturierenden Deutschland-Bildes können auch noch einmal aus einer anderen, neueren Sichtweise bestätigt werden. In Jurek Beckers (1937?-1996) weltweit anerkanntem Roman Jakob der Lügner (1969) liest der Pfannkuchenbäcker Jakob, der gleichsam aus Versehen in den Ruf geraten

\footnotetext{
${ }^{5}$ Dessen Erinnerung uns anlässlich der 2000-Jahr-Feier der Schlacht im Teutoburger Wald (wo immer się gewesen sein mag) auch noch im 21. Jahrhundert (dazu u.a. Wiegels \& Woesler, Hrsg., 2003) beschäftigt.
} 
ist, ein Radio zu besitzen und nunmehr, um den Lebenswillen der von den Nazis in ein Ghetto gepferchten Bewohner eines osteuropäischen Shtetls zu erhalten, immer neue Nachrichten vom Frontverlauf produzieren muss, erwartungsvoll eine deutsche Zeitung, die er auf einer Latrine gefunden hat. Ihm sind die Ideen schon längst ausgegangen, freilich stellen sich die Deutschen - im Spiegel dieser Latrinenzeitung auch nicht als besonders komplexe, reichhaltig angelegte Leute dar:

Jedenfalls hat ein Blick in das Blättchen genügt, und schon hat man gewusst, woran man war. Ständig haben sie sich bedroht gefühlt und erniedrigt und von Gott und der Welt benachteiligt, nicht sie haben uns erniedrigt, sondern wir sie. Die Frage, wie lange noch Deutschland unter den schmachvollen Ergebnissen des letzten Krieges leiden soll, hat ihnen in keiner Ausgabe Ruhe gelassen, dreimal die Woche. (Becker [1969], 1976, S. 113)

Becker selbst, der sich immerhin als deutschsprachiger Schriftsteller, Deutscher und Kind jüdischer Eltern verstand, kann auch deshalb hier angesprochen werden, weil sich in seinem Text Auto-Image und Hetero-Image, Eigenbeobachtung und Fremdbeobachtung ineinander verschränken, so wie dies im eigentlichen Sinne Thema und Einsatzpunkt einer interkulturellen Lektüre und Analyse in komparatistischer Sicht sein kann.

\section{Komparatistische Imagologie: Images/Mirages - Vorurteile und Stereotypen}

Wir kommen damit zum nächsten Punkt: Der begrifflich-theoretischen Arbeit in einem Feld von Bildern über andere Menschen, Gesellschaften und Völker, einer zumeist auf Texte bezogenen komparatistischen Imagologie, die aber ihrerseits sowohl angewiesen ist auf Kulturgeschichte und Soziologie, vergleichende politische Kulturforschung und die empirische Erforschung von öffentlichen Meinungen, Einstellungen und Wertsetzungen sowie deren kulturelle Grundlagen und Hintergründe als auch auf diese zurückwirken kann mit ihren eher an schriftlichen Texten, literarischen Quellen gewonnenen Befunden. Allen Polemiken zum Trotz, die sich zumal in den 1950er Jahren zwischen einer sogenannten ,amerikanischen“ Schule der Komparatistik, die etwa im Sinne René Welleks an der philologischen Erarbeitung von Texten ausgerichtet sein sollte, und einer „französischen“ ergaben (Dyserinck, 1981, 2. Aufl., S. 49ff.), stellen solche imagologisch-kulturwissenschaftliche Studien ein wichtiges, inzwischen auch in der Fachdisziplin anerkanntes Arbeitsfeld der Komparatistik dar (Corbineau-Hoffmann, 2000, S. 171ff.; Zima, 2003) und bieten, auch wenn sich die damit erwartete Aufwertung der zumindest in Deutschland immer noch als „kleines Fach“ gehandelten Komparatistik (Kiefer und Nell, 2005) nicht eingestellt hat, eine Reihe von Anschlussstellen an andere kulturwissenschaft- 
lich oder auch interkulturell ausgerichtete Arbeitsfelder und Studiengänge (Hofmann 2006, S. 51-60).

Auf die Vorläufer einer solchen auf Systematik, Vergleich und Verstehen ausgerichteten Betrachtung anderer Völker und der eigenen Gesellschaft - im Rückbezug auf die an anderen Literaturen und Blickrichtungen vermeintlich erkennbaren Bilder, die sich freilich auch in den Vorstellungen spiegeln, die diese anderen Gesellschaften sich von der jeweils eigenen des Betrachters machen - kann ich hier nicht genauer eingehen. $\mathrm{Zu}$ nennen sind aber Reiseberichte wie der des bereits erwähnten Herodot, zudem Texte wie die Abenteuer des Marco Polo (die Reise dauerte der Erzählung nach von 1271-1291), das Reisebuch des Ritters John of Mandeville aus dem frühen 14. Jahrhundert oder auch die „Reise um die Welt“ des arabischen Gelehrten und Juristen Ibn Battuta (1304-1368/1377?), die auch dann, wenn es sich um weitgehend erfundene Berichte handeln sollte - alle drei genannten stehen seit langem schon unter dem Verdacht, zumindest weitgehend „erfunden“ zu sein (Greenblatt, 1994) - doch immer noch einen Bildbereich zeigen, auf den bezogen sich in den folgenden Jahrhunderten Völker beschrieben und interpretiert fanden, obgleich sich die empirischen Bezüge entweder gar nicht oder nur in höchst zweifelhafter Weise belegen ließen. Offensichtlich gibt es eine, bereits in der hebräischen Bibel (die sogenannten „Völkertafeln“ in Genesis 1,10) nachweisbare, Topik hinsichtlich der Beschreibung bestimmter Völker, die sich dann, angereichert um die unterschiedlichsten Merkmale und Phantasmen, zu einem Katalog von Eigenschaften der Völker ausarbeiten ließ, der vom Spätmittelalter bis in die Neuzeit die Sichtweise der einzelnen Völker unter einander und so auch deren jeweilige Selbstinterpretation bestimmte (Koebner \& Pickerodt, 1987; Pochat, 1997). Dass die Entdecker und Eroberer der Neuen Welt und der anderen Kontinente und Völker diese auch noch in der Neuzeit in den Mustern der antiken Ethnographie und der an diese anschließenden Tradition sahen bzw. sehen wollten und entsprechend interpretierten, ist inzwischen hinreichend, u.a. bei Kohl 1982, dokumentiert.

Aber auch die Empirisierung der Völkerbeobachtungen, wie sie sich seit dem 17. Jahrhundert erkennen lässt, und wie sie dann im 18. Jahrhundert im Geiste der Aufklärung weitergeführt, im 19. Jahrhundert zum Arbeitsfeld einer zunehmend sich dem Positivismus als Wissenschaftstheorie und Methodenlehre annähernden Ethnographie und Ethnologie wurde (Blok, 1985), steht noch immer im Bann dieser auf eine bildhafte und umfassende Darstellung eines Volkes als einer in sich geschlossenen, homogenen Einheit bezogenen Erkenntnis- und Vermittlungsbemühungen. Mehr noch, diese werden gerade im 19. Jahrhundert in den Mustern sich vermeintlich objektiv und neuartig darstellender ,wissenschaftlicher“ Impulse, als die neben biologischer Evolutionstheorie v. a. auch Völkerpsychologie und nicht zuletzt Rassismus (Pollak, 1989) zu nennen sind, aufgenommen und weitergeführt. In beiden Bereichen, dem der empirischen Beobachtung und dem der verstehenden Anteilnahme an anderen Menschen, zumal auch in dem Bestreben, entsprechende Bilder 
anzufertigen und weiterzuführen, ist bereits seit dem Beginn der Neuzeit der Übergang zu den literarischen Formen fließend, zumal bis 1780 die Ausdifferenzierung eines eigenständigen Feldes des Fiktiven gegenüber einem anderen realitätsbezogenen, auf die Faktizität des gegebenen und Beobachteten ausgehenden Zugangs noch nicht stattgefunden hat - und auch in späterer Zeit nicht abgeschlossen ist (Nell, 2002, S. 101). Vielmehr ist es gerade so, dass mit dem ab 1760 in Europa sich abzeichnenden Feld einer autonomen „schönen“ Literatur auch für die Völkerbeschreibungen ein neues Feld erkennbar wird.

In dem Maße, in dem im Rahmen der sich erweiternden und differenzierenden bürgerlich-adligen Literaturen seit der zweiten Hälfte des 18. Jahrhunderts Bildungsund Entwicklungsprozesse, zumal im Roman und auch als Herzens- und Gefühlsbildungen (so in Laurence Sternes Sentimental Journey, 1768), in den Vordergrund treten, wird nicht nur erneut die Reise durch fremde Länder, sondern eben auch die Erfahrung und Schilderung der fremden Völker - sowohl für die Helden als auch für die Autoren, die über sie berichten - zu einem neuen Handlungs- und Bewährungsfeld. Dementsprechend verfolgte die nach 1945 auf den Trümmern Europas (neben Weltkrieg und Shoah war es in französischer und englischer Perspektive auch das Ende der europäischen Kolonialreiche, das sich abzuzeichnen begann) sich neu formierenden Komparatistik den Ansatz, in der Arbeit an diesen Bildern sowohl etwas über die Eigenart und die Funktionen literarischer Texte herauszufinden, als auch tatsächlich durch die vergleichende Arbeit an den verschiedenen Images/images zum Wissen der Völker übereinander und zu ihrer Verständigung beizutragen. Den Grundstein zu einer solchen Imagologie legte der französische Komparatist MariusFrançois Guyard (1921-2011) mit dem inzwischen berühmten achten Kapitel seiner in der populären Reihe Que sais-je? 1951 erschienenen Einführung La Littérature Comparée, das dort den Titel: L'étranger tel qu'on le voit trägt und den Grundriss einer Arbeit an den Bildern des anderen in den Medien der Literatur entwirft (Guyard, 1951, S. 110-119).

Waren diese Ansätze zunächst stark und noch bis in die 1970er Jahre hinein davon ausgegangen, dass es sich bei Völkern um einigermaßen homogene Großgruppen handeln würde, deren Weltwahrnehmung und Einstellungen (Mentalität), kulturelle Stile und Habitus-Formen tatsächlich in sich stimmig genug seien, um gegeneinander abgehoben und in diesem Sinne dann miteinander vergleichbar erscheinen zu können, so hat die neuere insbesondere soziologische, ethnologische und kulturhistorische Forschung (auf Georg Simmel als Vorläufer wurde ja schon hingewiesen) inzwischen gerade diese Einheitlichkeit der Volkscharaktere, einer entsprechenden Volksseele - die nun auch auf der Basis der aktuellen Genforschung als widerlegt gelten können (Cavalli-Sforza, 1994) - und weitere Allgemeinplätze in Frage gestellt. Vorstellungen und Beobachtungen über die Natur bzw. auch die Seele oder den „Geist der Völker“, die von Herder und anderen Autoren des 18. Jahrhunderts entwickelt worden waren, verfestigten sich im Zusammenhang des Aufstiegs 
biologistischer Denkmodelle und Erklärungen (Mann, 1973) zu dann rassetheoretisch begründeten Kulturgeschichten und anthropologischen, in gewissem Sinne auch religionsähnlichen Spekulationen (Mosse, 1990, S. 111-135; Clausen, 1994, S. 92-94), die freilich wieder auf die Literatur- und andere Geisteswissenschaften Einfluss hatten (Bermbach, 2015, Münkler 2015), allerdings auch schon nach der Jahrhundertwende begründete (und bis heute aktuelle) Kritik erfuhren (Hertz, 1904). Friedrich Otto Hertz (1878-1964, seit 1946 Frederick Hertz), der seit Ende der 1920er Jahre bis zu seiner Vertreibung durch die Nazis 1933 in Halle Soziologie lehrte (Pasternak \& Sackmann, 2013, S. 55-76), kann aber nicht nur als einer der ersten Kritiker eines aktuell wieder im Schwange stehenden Rassismus gesehen werden. Vielmehr bieten seine Schriften, etwa der immer noch lesenswerte Beitrag zum Stichwort „Rasse“ im 1931 von Alfred Vierkandt herausgegebenen Handwör terbuch der Soziologie (Hertz, 1931), auch ein Beispiel für den Stand soziologischen und kulturgeschichtlichen Forschens in Deutschland vor 1933 und den durch die Nazi-Zeit, zumal die Vertreibung jüdischer Gelehrter eingetretenen Verlust (Nell, 2013).

Wenn vor diesem Hintergrund aktuell in der Imagologie, einem außer in der Komparatistik auch in der Soziologie schon seit Längeren, wenn auch nicht so stark beforschten Feld (Dreitzel, 1962), das derzeit mitunter recht unreflektiert und gegen die eigenen Intentionen standardisierend in verschiedenen interkulturellen Ansätzen fröhliche Urstände feiert (Thomas, 2005; Hofstede, 1991), mit Images/mirages gearbeitet wird, so müssen diese Bilder immer in einem doppelten Sinne als Konstrukte gesehen werden: Nicht nur dass sie als „semantisches Differential“ (Osgood, Suci \& Tannenbaum, 1957) erhoben und dann dazu genutzt werden können, die Einstellungen der Beobachter zu erkennen und zu interpretieren. Vielmehr lassen sich auch mit Blick auf die in Betracht gezogenen Länder bzw. Völker bestimmte Setzungen und Verhaltensmuster der Beobachter erkunden, freilich immer auch nur im Bewusstsein, dass es sich um ein konstruiertes Bild handelt. „Das grundlegende Merkmal des Bildes vom anderen Land“", so der in den USA lehrende Germanist und Komparatist Peter Boerner in einer einschlägigen Studie,

\footnotetext{
ist, dass es reflektiert, wie ein Volk oder wie repräsentative Teile dieses Volkes von anderen Völkern, insbesondere von deren prominenten Vertretern gesehen werden. Da sich dieses „Sehen“ notwendigerweise aus vielen Einzelbeobachtungen, Meinungen und subjektiven Bewertungen zusammensetzt, ist jedes Bild vom anderen Land letzten Endes ein verwickeltes Konglomerat (...) oder eine Hilfskonstruktion, in der alle einschlägigen Beobachtungen und Reflexionen zusammengefasst sind. Die Möglichkeit, sich dieses Konglomerat zu vergegenwärtigen, beruht auf der Zuversicht, dass die angenommenen Charakteristika des beobachteten Volkes in der Vorstellung eines wesentlichen Teils des beobachtenden Volkes ein definierbares und beschreibbares Bild ergeben. Verschiedene Bilder eines einzelnen Volkes können nebeneinander bestehen oder sich ablösen, je nach ihren Ursprüngen in bestimmten Teilen des Lesepublikums oder je nach ihrer Entwicklung über einen bestimmten Zeitraum hinweg. (Boerner, 1975, S. 30)
} 
Was hier reflektierend eingegrenzt und gerade dadurch in seiner Objektivität bzw. in seinen Erkenntnisansprüchen sowohl produktiv geöffnet als auch abgesichert werden sollte, scheint durch die ethnologische Reflexion und konstruktivistische Wissenschaftskritik der letzten Jahrzehnte weitergeführt und zugleich aufs Neue in Frage gestellt zu sein (Cappai, Shimada \& Straub, 2010). Gerade im Anschluss an die in der nordamerikanischen Ethnologie/Social Anthropology seit den 1960er Jahren geführte Debatte um „Observers observed“ (vgl. Berg \& Fuchs, 1993) können solche Bilder von anderen Ländern und Völkern wohl eher dazu herangezogen werden, Vorstellungen und implizite Setzungen der Beobachter zu erkunden, als die angebotenen Bilder als Wirklichkeitsaussagen im Hinblick auf die zu schildernden Gesellschaften zu interpretieren (vgl. dazu Hofmann, 2006, S. 36ff.). Gleichwohl muss in der Existenz und Ausstrahlung der images doch auch eine Evidenz bzw. Objektangemessenheit hinsichtlich der beobachteten Gesellschaft und ihrer Erscheinungsformen mit in Rechnung gestellt werden. Zum einen lässt sich das am Gebrauchswert, also beispielsweise an den Verkaufszahlen, entsprechender Reiseführer ${ }^{6}$ ablesen, zum anderen daran, dass Autoren, wenn sie denn den Bezug auf eine bestimmte Gesellschaft suchen, sei es die eigene, sei es eine andere, doch auch bestimmte Plausibilitäts- und Evidenzmerkmale setzen und vermitteln müssen, um die in Anspruch genommenen Bezüge tatsächlich glaubwürdig zu machen. Dass in diesen Zusammenhängen, nicht zuletzt in Verbindung mit dem fiktionalen Status literarischer Texte, Images/Mirages den Status von Kippfiguren haben, also sowohl als Spiegel als auch als Glas genutzt werden können, einmal um durchzuschauen, einmal um sich selbst „im Spiegel“ der anderen sehen zu können, erhöht ihren Reiz und ihre Komplexität. Wenn sie darauf hin entwickelt werden und nicht einfach als Ausdruck von Vorurteilen oder als Stereotypen (feststehende Wechselbilder, kognitive Dissonanz) fungieren, macht dies sicherlich die Arbeit mit ihnen nicht einfacher: Ist die „schöne Polin/Jüdin“ nun eine Form der Aufwertung oder Abwertung? In welchem Sinn lässt sich die „schöne Frau Seidenmann“ in Andrzej Szczypiorskis gleichnamigem Roman und Bestseller (Szczypiorski, 1988) als ein Beitrag zur Schilderung von einer weiblichen Gestalt im Allgemeinen, einer deutschen, polnischen... usw. Frau im Besonderen verstehen? (Grözinger, 2003; Orłowski, 2005). Anders als zunächst in den 1960er Jahren in fachinnovativer Hinsicht erwartet, stellt die Imagologie also weder einen genuin literaturwissenschaftlichen Arbeitsansatz noch einen Königsweg zur interkulturellen Verständigung dar, sondern bedarf selbst durchaus deutlicher Historisierung und theoretischer Einbettung sozial- oder kulturtheoretischer Art, wobei eine soziologische Fundierung etwa im Anschluss an Erving Goffmans Rahmenanalyse (Goffman, 1977) oder phänomenologisch grun-

\footnotetext{
${ }^{6}$ Vgl. dazu den Verkaufserfolg, die Beachtung und Kritik, die etwa die populären „Führer“ durch die polnische Gesellschaft und Geschichte von Steffen Möller oder Radek Knapp gefunden haben (Möller, 2008; Knapp, 2005).
} 
dierte Konzeptionen der Fremdwahrnehmung im Anschluss an Husserl (Waldenfels, 2006) auch forschungspraktisch am genauesten und zugleich aussichtsreichsten erscheinen dürften.

\section{Deutschlandbilder}

In Anlehnung an den französischen Philosophen und Wissenschaftshistoriker Étienne Balibar, der einen „Willen zum Wissen“ und einen „Willen zur Macht“ und in der Folge die Macht der „Verkennung“ als grundlegende Motive des modernen Rassismus bestimmt hat (Balibar, 1989), ließe sich im Blick auf die durchaus älteren Völkerbilder ebenfalls von einem „Willen zum Wissen“ und einem Bedürfnis nach Klassifikation und Übersichtlichkeit, unter der Berücksichtigung ihrer Möglichkeiten und Gefährdungen durch Verformung und Verkennung sprechen. Aus der Perspektive von Reisenden und Kaufleuten, die neben Unterhaltung aus den Berichten über fremde Völker sicherlich auch Orientierung und Ratschläge zum Verhalten erwarteten, kann außerdem wohl auch ein Bedürfnis nach Sicherheit auf der Basis angemessener und zutreffend interpretierter Information angenommen werden. Damit war es (ist es?) allerdings mitunter nicht weit her, wie sich am Beispiel einer der in der ersten Hälfte des 18. Jahrhunderts durchaus beliebten, zumal in pädagogischen Zusammenhängen gerne verwendeten Völkertafel zeigen lässt. ${ }^{7}$

Auch sind in einer solchen, durchaus sich sachlich gebenden Übersicht natürlich auch der Standpunkt der Betrachter und die sie beherrschenden Diskurse zu erkennen, besonders dort, wo über Wertsetzungen oder Glaubensbekenntnisse gehandelt wird. Tatsächlich aber sind zumal als literarischer Stoff die Völkerbilder schon älter und variieren auch mit Blick auf Deutschland bis zur zweiten Hälfte des 19., ja bis zum Beginn des 20. Jahrhunderts eigentlich überraschend wenig. Die Deutschen werden zwar nicht besonders geschätzt, gelten aber eher als harmlos und unpolitisch. Die Boshaftigkeit und Machtgier, eine Eigenschaft, die schon im 18. Jahrhundert an Preußen bemerkt worden war, ist wie die deutsche Selbstzuschreibung als Kämpfer und Soldat tatsächlich so jung wie der nationalistische Wahn im Ganzen auch: Geboren im Umfeld der Romantik, zugespitzt bzw. schlagkräftig geworden in der durchaus kulturrevolutionär und im modernen Sinn ideologisch bewehrt auftretenden Begeisterung der Befreiungskriege, zu einer Art Staatsdoktrin erhoben im Zweiten Kaiserreich und als kulturell-politisch fundiertes Wahnsystem dann zum Bildungsgut und zur Ideologie der bürgerlich-adligen und kleinbürgerlichen Schichten, ja, was die Führung der Sozialdemokratie angeht, auch der Arbeiterbewegung, geworden. ${ }^{8}$

\footnotetext{
${ }^{7}$ Die inzwischen vielfach beachtete Tafel stammt wohl aus der ersten Hälfte des 18. Jahrhunderts aus der Steiermark (Stanzel, 1998). Eine Abbildung findet sich u.a. bei Wikipedia: Völkertafel.

${ }^{8}$ Für eine mikrosoziologische Untersuchung und Kulturgeschichte einer ebenso einschlägigen wie markanten Geste: Allert, 2010.
} 
So wie dies der in Stanford lehrende Historiker Eugen Weber für Frankreich in den Jahren nach 1870 beschrieben hat (Weber, 1976), kann dieser Prozess auch für andere europäische Gesellschaften, zumal die deutsche/preußische Gesellschaft beschrieben werden (Johnston, 1990). Die eigentliche Zeit der Nationalbewusstseinsbildung, der Schaffung der Nation und auch der Fundierung dieser Nation in den entsprechenden Mythen und Leitbildern ist nach den romantischen Anfängen die zweite Hälfte des 19. Jahrhunderts (so auch in Mittelosteuropa). Es nimmt daher nicht wunder, dass zumindest zwei bekanntere Deutschen-Bilder: der ,deutsche Michel“ ebenso wie der „deutsche Militär", in eben dieser Zeit entstanden bzw. populär geworden sind, und dass sich andere, etwa der ,gemütliche Deutsche“ oder auch der Deutsche als „Nazi“ gegenüber diesen beiden auch weniger universal einsetzen lassen, wenn es darum geht, ein Bild „der“ Deutschen zu entwerfen (Rösgen, 1999; eine Gegenüberstellung dieser bekannten „Deutschen“-Bilder findet sich auf der Titelseite des Spiegel vom 22. Juli 1964).

Bemerkenswert ist auch, dass dies die Zeit ist, in der sich - verbunden mit den Nationalstaatsprojekten und ihren Ideologien - die einzelnen Bilder: Hetero-Images und Auto-Images in vielen Nationalstaats-Bildungs-Projekten (Anderson, 1988) formieren und durch die entsprechenden Transmissionsriemen bis ins letzte Dorf verbreitet werden: Schule und Militär, Publizistik und politische Propaganda, für Deutschland besonders die scheinbar unpolitische Organisation der Zivilgesellschaft im Vereinswesen: in Turn-, Gesangs- und Heimatvereinen; Kriegerdenkmäler und Feiertage von der Art des Sedanstags tun ein Übriges dazu wie Turnvater-JahnStraßen, die es bis heute gibt; ebenso (und immer noch) werden das Lob des deutschen Waldes (oder die Sorge um ihn), deutsche Treue, deutsche Frauen, deutscher Wein und deutscher Sang, auch noch andere „goldene Werte“ aufgeboten, wenn es darum geht, den Konturen des 1871 neu entstandenen Deutschen Reiches eine gefühlsbasierte Grundlage zu geben. Hier, also am Vorabend des 20. Jahrhunderts, findet die eigentliche Formierung der auf eine vermeintlich national, und dies meint dann eben auch „rassisch“, reine Gesellschaft gegründeten Nationalitätsvorstellungen statt, die dann im Spiegel der Bilder der anderen und von sich selber gestaltet, befragt und reflektiert werden könnte; auch der komparatistische, Kulturen als Nationalkulturen vergleichen wollende Ansatz hängt noch immer an dieser langen Kette.

Freilich herrscht mitunter noch immer, zumal in angeheizten, oftmals innenpolitisch motivierten politischen Debatten, aber auch in Unterhaltungsformaten, das Bild der Deutschen von Nazis und sonstigen Schurken der 1930er und 1940er Jahre vor. Gleichwohl ist auch darauf hinzuweisen, dass sich dieses Bild der Deutschen als einer machtversessenen und militaristisch ausgerichteten Gesellschaft (Seib \& Koch, 1989) tatsächlich erst durch die Überdeckung einer reichhaltigeren deutschen Geschichte und Gesellschaft mit dem Aufstieg Preußens seit dem 18. Jahrhundert und dann durch eine im Geiste des Hohenzollerntums nach 1871 nachdrücklich in die deutschen Mentalitäten und Landschaften hineingebrachte Ideologie des Krie- 
gers und der Kampfbereitschaft formiert hat. Dass hierzu ein älterer Wille bzw. auch eine vorgängige Nötigung zur Unterwerfung unter „Vormünder“ (I. Kant) beigetragen hat, eine wohl durch Protestantismus und Pietismus, Kleinstaaterei und katholische Bevormundung geförderte Ohnmacht und Unmündigkeit der bürgerlichen Gesellschaft (vgl. Mayer, 1984), ist ebenfalls zu konstatieren.

Tatsächlich konnte um 1900, wie es Heinrich Mann und Norbert Elias gleichermaßen beobachtet haben, der preußische Leutnant ein gesamtgesellschaftliches Leitbild bieten (Elias, 1989, S. 67-71), ja „es gab“, wie Christian Graf von Krockow Theodor Mommsen zitiert, „Todesanzeigen von hochverdienten Professoren aus der Kaiserzeit, die ihre Stellung als Leutnant der Reserve noch vor ihrer Mitgliedschaft in hohen Akademien der Wissenschaften angaben" (von Krockow, 1995, S. 114). Vor dem Hintergrund des Ersten Weltkriegs notierte der zeitweilige französische Premierund Kriegsminister Georges Clemenceau (1841-1926), zugleich 1919 in Versailles Verhandlungsführer der französischen Regierung gegen die deutschen Interessen:

(...) es entspricht dem Wesen des Menschen, das Leben zu lieben. Der Deutsche kennt diesen Kult nicht. Es gibt in der deutschen Seele, in der Gedankenwelt und Literatur dieser Leute eine Art Unverständnis für alles, was das Leben wirklich ist, für das, was seinen Reiz und seine Größe ausmacht, und an dessen Stelle eine krankhafte und satanische Liebe zum Tod. Diese Leute lieben den Tod. (...) Woher haben sie das? Ich weiß darauf keine Antwort. Der Deutsche liebt den Krieg als Selbstliebe und weil an dessen Ende das Blutbad wartet. (von Krockow, 1995, S. 26f.)

Diese Bemerkungen spielen zunächst auch auf die seit den ersten Jahrzehnten des 19. Jahrhunderts sich verbreitende Nibelungen-Mythologie an. Deren Ausstrahlung wurde seit den 1870er Jahren nicht nur durch die Faszination für Richard Wagners Ring des Nibelungen (1851-1874; 1876) gesteigert, sondern konnte dann auch zu einem Sinnbild für jene „Lust am Untergang“ (Münkler \& Storch, 1988) werden, die sich im Rückblick auf die Zeit des Nationalsozialismus und ihre Vorgeschichte als eine Spur der Nekrophilie innerhalb der deutschen Kultur der Jahre zwischen 1871 und 1945 bestimmen lässt (Friedländer, 1984). Freilich geben sie nicht das ganze, wesentlich umfangreichere Deutschland-Bild der Älteren wieder, auch nicht die Grundtendenzen gegenwärtiger Deutschland-Beobachtungen und -Bilder (dazu jetzt Orłowski \& Karolak, 2015). Vielmehr zeigt sich in den älteren Texten zunächst einmal eine den Freuden des Lebens sogar recht zugewandte Seite der Deutschen, wobei allenfalls die Vernachlässigung geistiger Bedürfnisse auffallend erscheint: Freude an Wissen und Gesprächen sehen ausländische Beobachter offensichtlich ebenso wenig wie das Interesse an gemeinschaftlichem Räsonieren und an Gesprächsspielen von der Art der in Frankreich, aber ebenso bereits im Italien des Quarto- und Cinquecento bekannten Hof- und Salonliteratur, in der insbesondere auch Frauen den Ton angaben.

Das ältere Deutschland-Bild wird noch immer ganz zutreffend in einem noch heute lesenswerten, wegen seines penetranten Antisemitismus allerdings nicht gera- 
de als Lesevergnügen einzustufenden Schelmenroman aus der Shakespeare-Zeit auf den Punkt gebracht. In Thomas Nashes (1567-1601?) 1594 erschienenem Roman The Unfortunate Traveller werden im Rückgriff auf ältere Topoi auch Anweisungen zum Reisen gegeben, freilich zum einen unter dem Vorbehalt, dass das Reisen insgesamt von Übel sei: „The first traveller was Cain, and he was called a vagabond runagate on the face of the earth" (Nashe, 1985, S. 341), zum anderen sind - ironisch genug - die Schelme ja sowieso schon unterwegs, auf der Flucht vor der Bestrafung, hatten also gar keine andere Wahl als mobil zu sein. In diesen Zusammenhang gehören dann Hinweise auf die Nutzlosigkeit des Reisens in die verschiedenen europäischen Länder und so auch nach Deutschland.

With the Dane and the Dutchman I will not encounter, for they are simply honest men, that ... do nothing but fill bottomless tubs and will be drunk and snort in the midst of dinner. He hurts himself only that goes thither; he cannot lightly be damned, for the vinters, the brewers, the malt-men, the alewives pray for him. 'Pitch and pay, they will pray all day.' (Ebd., S. 345) ${ }^{9}$

Ähnlich, aber ein wenig menschenfreundlicher und weniger grotesk, erscheinen die Deutschen in Honoré de Balzacs (1799-1850) Novelle L'auberge rouge von 1831; hier wird Hermann, der deutsche Freund eines Pariser Bankiers, so geschildert:

\footnotetext{
Dieser Freund, der Inhaber einer bedeutenden Nürnberger Firma, war ein biederer, dicker Deutscher. Er verfügte über Geschmack und Bildung und war ein leidenschaftlicher Pfeifenraucher; er hatte ein hübsches, breites Nürnberger Gesicht und eine viereckige, offene Stirn, die von spärlichen blonden Haaren umrahmt war. Er war der echte Sohn des edlen und reinen Germanien, das so fruchtbar ist an ehrenwerten Charakteren, deren friedfertige Sitten sich selbst nach sieben Invasionen nicht verleugnen. Der Fremde hat (sic!) ein argloses Lachen, hörte aufmerksam zu und trank sein gehöriges Quantum; ... Er hieß Hermann wie fast alle Deutschen, die uns von Schriftstellern geschildert werden. Als Mensch, der nichts leichtzunehmen weiß, saß er behäbig am Tisch des Bankiers und speiste mit dem in Europa so berühmten altdeutschen Appetit. (von Krockow, 1995, S. 27f.)
}

Ein genauerer Vergleich der bislang zitierten, auf den ersten Blick doch recht unterschiedlich wertenden Darstellungen würde allerdings einige Gemeinsamkeiten herausstellen, die sich bis zum 20. Jahrhundert in den Bildern von den Deutschen im Medium anderer Betrachter und ihrer literarischen Gestaltung finden lassen: Ruhe und Gefräßigkeit, Trinkfestigkeit und „,deutsche Gemütlichkeit“; kaum geistige Ansprüche, von Eleganz ist ebenso wenig die Rede wie von Gesellschaftssinn; dafür aber Treue und Tiefsinn, insgesamt eine gewisse Beschränktheit, in der sich sogar die Deutschen selbst, zumindest seit der politisch aufgeladenen Romantik, zeitweise gut getroffen fanden, wie die Popularität dieses immer wieder einmal aufgetischten Sprichworts belegen kann: „Deutsch sein heißt, eine Sache um ihrer selbst willen

\footnotetext{
${ }^{9}$ Möglicherweise ist hier auch Neid mit im Blick, wie auch der Roman im Ganzen durchaus menschenfeindliche und ressentimentgetragene Beobachtungen zusammenstellt.
} 
tun" - wurde von Fichte über Wagner bis zu Goebbels gerne als Ausweis einer spezifisch „deutschen“ Tugend zitiert. (Aber ist es überhaupt eine Tugend, eine Sache immer nur und lediglich um ihrer selbst willen zu tun?)

Will man Deutschlandbilder (schon dass es ein Plural ist, muss betont werden) in einem großen Bogen zusammenfassen, ${ }^{10}$ so lassen sich folgende Gesichtspunkte herausarbeiten und zumindest als Thesen formulieren:

1. Bis weit ins 19. Jahrhundert hinein stellen die Deutschen für die meisten ausländischen Betrachter weniger eine Bedrohung dar als vielmehr das Beispiel einer weitgehend in der Vormoderne, in einer Gegenwelt zur jeweils aktuellen oder modernen Zivilisation lebenden ländlich-kleinstädtischen Gesellschaft. Tatsächlich handelt es sich weitgehend um die Beschreibung einer „Männerwirtschaft“, in der tüchtiges Essen und Trinken auf der Tagesordnung stehen, auch Händel und Raufereien; bieder, tumb und treu stellen sie sich dar, die „alten“, die „Ur-“Deutschen, bereits zum Hintersinn fehlt ihnen weitgehend der Geist, von anderen Vergnügungen des Denkens einmal ganz zu schweigen.

2. Diese Sicht der Deutschen - bei Tacitus (98 n. Chr.) wurden so bereits die Germanen geschildert (Tacitus, 1971, S. 18) - stellt nun wie alle Images ein nach mindestens zwei Seiten hin ausdeutbares Bild dar: Waren den einen Kampfbereitschaft und mangelnde Reflexionsfähigkeit eine Tugend, so konnten beide Beobachtungen den anderen als Beispiele mangelnder Zivilisiertheit, ja Zivilisationsunfähigkeit gelten. Die Selbstbegründung einer gewünschten Vorrangstellung der eigenen Kultur und Gesellschaft aus dem Abstand zu den Deutschen konnte hier ebenso ihre Grundlage finden wie die Kritik der eigenen Gesellschaft mit dem Verweis auf die „Geburt der Freiheit in den germanischen Wäldern“, mit der Montesquieu (1689-1755) im Rahmen seines Nachdenkens über den Geist der Gesetze (De l'ésprit des lois, 1748) aufwartete, oder später Mme de Staël (1766-1817), die gegenüber dem modernen, „rational“ planenden Macht- und Verwaltungsstaat, wie sie ihn durch die Politik ihres Gegners Napoleon verwirklicht sah, das romantische Deutschland als das Land der Nebel und der Wälder, der kleinen Städte und alten Handwerke, zumal aber als das Land der Philosophen und Musiker für sich entdeckte, ja in gewissem Sinne erfand (De l'Allemagne, 1813). Ein Bild, das sich auch im 20. Jahrhundert in Frankreich noch großer Popularität erfreute und in den literarischen bzw. filmischen

\footnotetext{
${ }^{10}$ Tatsächlich müssten unterschiedlichste Differenzierungen angebracht werden: nach Zeiten und Ländern, nach der sozialen Differenzierung und den unterschiedlichen Nähe- bzw. Kontaktchancen der einzelnen Betrachter-Gruppen zu den jeweils in den Blick genommenen Länder- bzw. Kulturbezügen, schließlich, wie auch schon angesprochen, historische und politische, soziale und kulturelle Rahmensetzungen und konkrete Ereignisse, dann auch, zumal seit 1800, die Publizistik, die Medien und Mediensysteme, Schulbücher, Reiseführer, Unterhaltungs- und Schemaliteratur, und nicht zuletzt Veränderungen im Selbstverständnis und in den Vorstellungswelten der Funktionseliten, generationen- und geschlechterspezifische Unterschiede.
} 
Werken von Romain Rolland, Jean Giraudoux, Henri-Pierre Roche/François Truffaut oder auch Michel Tournier seinen Niederschlag gefunden hat.

3. Komplementär zu dieser Außensicht entwickelten die Deutschen - in ihren verschiedenen Landschaften, konfessionellen und religiösen Grundierungen, kulturellen und sonstigen sozialen Differenzierungen - natürlich auch ihre eigenen Bilder von sich, wobei hier ebenfalls Orientierung und Differenzsetzung gegenüber den anderen den Ausschlag gaben. Dazu hatten die Humanisten, Luther und Ulrich von Hutten, Melanchthon und später auch die Poeten und Poetologen des 17. Jahrhunderts (sprachpflegerische Gesellschaften; „à la mode“-Kritik) schon einen Vorlauf erbracht. Im Rahmen der sich seit dem 18. Jahrhundert durchsetzenden Nationalstaats- und Nationalkultur-Projekte der Bildungs- und Wissenschaftseliten wurde nunmehr eine Plattform für die Ausbildung eines deutschen Selbstverständnisses erstellt, auf der sich im Selbstbild freilich protzende Aufdringlichkeit und - historisch erzeugt durch Militarismus und religiösen Fanatismus - ein Gefühl ewiger Unterlegenheit und Benachteiligung in ebenso nachhaltiger wie unangenehmer Weise mischten. ${ }^{11}$ Im Schatten des gerade eben begonnenen Ersten Weltkriegs erhob der Dramatiker Ludwig Fulda den Anspruch auf einen

\begin{abstract}
deutschen Shakespeare: Unser Shakespeare, so dürfen wir ihn nennen, wenn er auch versehentlich in England zur Welt kam. So dürfen wir ihn nennen mit dem guten Recht der geistigen Eroberung. Und falls es uns glückt, England niederzuzwingen, dann meine ich, wir sollten in den Friedensvertrag eine Klausel setzen, wonach William Shakespeare auch formell an Deutschland abzutreten ist. (zitiert nach Hortmann, 2001, S. 19)
\end{abstract}

Eine solche maßlose Haltung machte natürlich auch vor den eigenen kulturellen Leistungen nicht halt: 1942 schrieb der Bonner Universitätsprofessor Adolf Dyroff in Aurora, der Zeitschrift der Eichendorff-Gesellschaft:

Wenn Eichendorff heute noch lebte, würde er, das ist sicher, mit heißem Atem sich einem der drei Wehrmachtsteile angeschlossen haben. Er würde im jähen Zusammenbruch Frankreichs eine gerechte Strafe für Ungerechtigkeit und Siegerübermut erkennen. Er würde mit starker Verbissenheit auf die Niederringung des perfiden Albion warten. (Zitiert nach Klausnitzer, 1999, S. 598) ${ }^{12}$

Militarismus und Kadavergehorsam sowie die Unfähigkeit zur Freude an einem autonomen, in individueller Selbstbestimmung natürlich auch sich begrenzen und reflektieren könnenden eigenen Selbst, um hier Theodor W. Adorno anzuführen (Adorno, 1969, S. 87-99), bilden in diesem Sinn die Grundlage einer Nekrophilie, die sich nicht nur in den Schlachtengesängen des Ersten Weltkriegs und in den seinerzeit viel gelesenen Erzählungen eines Walter Flex (1887-1917) niederschlagen.

\footnotetext{
${ }^{11}$ Wer hier mehr lesen will, sollte Herders Journal meiner Reise im Jahr 1769 oder auch Karl Mays Abenteuerroman Der blaurote Methusalem (1888/1889) lesen; zu Herders Reise Nell, 2016a.

${ }^{12}$ Allenfalls hört man hier noch das Gebiss am Bonner Schreibtisch klappern; mit Eichendorff, zumal seinem heutigen Verständnis, hat das gottseidank gar nichts zu tun.
} 
Statt zivilgesellschaftlichem Bürgerbewusstsein produzieren die Jahre nach 1871 tatsächlich einen Stolz auf die eigene Hässlichkeit. Demgegenüber bietet ein Blick auf die vorangehenden langen Jahrhunderte eine sehr viel differenzierter sich selbst wahrnehmende und von den anderen wahrgenommene Gesellschaft der Deutschen, differenziert nach Landschaften und Kleinstädten, Konfessionen und Herrschaften; es ist allerdings bemerkenswert, wie umfassend, nachhaltig und im wahrsten Sinn des Wortes „mörderisch“ diese Selbstprogrammierung der Deutschen noch bis in die 70er Jahre des 20. Jahrhunderts gewirkt hat (vgl. von Krockow, 1999).

4. Diese Selbstprogrammierung auf Hässlichkeit hatte natürlich ihre Stufen, beginnend mit den Befreiungskriegen (Kleists Die Hermannsschlacht, 1808), der Preisgabe des Liberalismus zugunsten des Nationalismus nach 1848, schließlich der wirklich mythopoetischen, also ideologischen Schaffung eines nationalistischen deutschen Selbstbildes, das dann als Korrelat übersteigerter nationalistischer Strömungen und politischer Programme auch in den anderen europäischen Ländern in die Schützengräben des Ersten Weltkriegs geführt hat (Nell, 2016b). Der auf höchstem technischen Niveau, nach rationalen Kriterien organisierte und mit zivilisatorischem „Anstand“ geplante und dann in die Tat umgesetzte Massenmord an einem ganzen europäischen Volk, wie ihn Nazi-Deutschland an den europäischen Juden begangen hat, brachte eine Färbung des Bildes mit sich, die als Tönung der deutschen Geschichte wohl erkennbar bleibt, bleiben wird und wohl auch bleiben sollte, da sie, ernst genommen mit der Gründung der Bundesrepublik Deutschland, unter tätiger, bis heute dankenswerter Hilfe des Auslandes, die Chance einer neu begründeten Bürgergesellschaft geschaffen hat. Dass auch diese Gesellschaft, die neben der Einigkeit vor allem Recht und Freiheit zu ihren Grundprinzipien erhoben hat, nicht ultrastabil beschaffen ist, sondern gefährdet bleibt und engagierte und selbstbewusste Bürger braucht, die für sie eintreten, in ihr leben wollen und dies auch (mitunter) gerne tun, mag so am Ende dieser Übersicht stehen.

\section{Perspektiven}

Mit Blick auf die Fülle der angebotenen Materialien seien hier nur zwei Punkte angeführt: Zum einen: Deutschlandbilder sind Wechselbilder und Kippfiguren: „Je näher man es anschaut, desto ferner schaut es zurück“, heißt hierzu das Motto, das Alexander Kluge im Rahmen seines Nachdenkens über die Geschichte Deutschlands und der Deutschen gewählt hat (Kluge, 1979). Die durch diese Schwankungen entstehende Distanz bietet einen Platz für Analyse und Selbst-Reflexion eben im Spiegel der anderen, ein Bedingungsverhältnis und eine Arbeitsaufgabe, die für das Leben in einer komplexen, modernen, offenen Bürgergesellschaft ebenso unverzichtbar wie unabschließbar ist. Zum anderen: Identitätsfragen sollten ebenso wie die Thematisierung kultureller Unterschiede, von denen Geoffrey Hartman sagt, sie 
„sollten stets Anlass von Komödien, nicht von Tragödien sein“ (Hartman, 1996, S. 199), nicht im Vordergrund individueller und sozialer Selbstverortung stehen bzw. stehen müssen. „Identität“, so ein Beobachter der aktuellen gesellschaftlichen Entwicklungen und Diskurse im Deutschland des Jahres 2016, „ist eine Vokabel, mit der sich ökonomische und politische Rivalitäten kulturalistisch verschärfen lassen“ (Günther, 2016). Der Bedarf an Identität wächst im Maße der Verunsicherung durch soziale und politische Veränderungen, er kann aber auch als Sprengsatz geschärft und als Brandbeschleuniger für unterschiedlichste Machtstrategien genutzt werden. Deutschlandbilder bieten hier in dem Sinne, in dem Klaus Eder von Kultur im Ganzen als einem Diskursfeld spricht, das durch die Erzeugung und Gestaltung von Dissenz an der Herstellung und an der Weiterführung eines gesellschaftlicher Konsenses mitwirkt (Eder, 1999, S. 149f.), ein Reservoir an Bildern, Projektionen und Erfahrungen zu weiterer diskursiver Auseinandersetzung (und damit auch Kommunikation) an. Ihr Nutzen und Anstoß kann darin bestehen, möglichst viele Stimmen zu Wort kommen zu lassen und damit Selbstbestimmung und selbstreflexive Möglichkeiten der Verortung (nicht nur im Ernst, sondern vor allem im Spiel $)^{13}$ zu ermöglichen.

\section{Bibliographie}

Adams, H. (1953). Die Erziehung des Henry Adams. Von ihm selbst erzählt. Zürich: Manesse.

Adorno, T. W. (1969). Erziehung nach Auschwitz. In ders. Stichworte. Kritische Modelle 2 (S. 83-101). Frankfurt a.M.: Suhrkamp.

Allert, T. (2010). Der deutsche Gruß. Geschichte einer unheilvollen Geste. Stuttgart: Reclam.

Anderson, B. (1988). Die Erfindung der Nation. Zur Karriere eines erfolgreichen Konzepts. Frankfurt a.M.: Campus.

Aristoteles (1995). Philosophische Schriften in sechs Bänden. Bd. 5: Metaphysik (Nach der Übersetzung von Hermann Bonitz, bearb. Horst Seidl). Hamburg: Meiner.

Balibar, E. (1989). Gibt es einen „,neuen Rassismus“? Das Argument, 175, 31. Jg., S. 369-380.

Barnes, J. (1989). Flauberts Papagei. Zürich: Haffmans.

Bausinger, H. (2000). Typisch deutsch. Wie deutsch sind die Deutschen? München: C. H. Beck.

Beck, U., Giddens, A. \& Lash, S. (1996). Reflexive Modernisierung. Eine Kontroverse. Frankfurt a.M.: Suhrkamp.

Beck-Gernsheim, E. (1999). Juden, Deutsche und andere Erinnerungslandschaften. Im Dschungel der ethnischen Kategorien. Frankfurt a.M.: Suhrkamp.

Becker, J. (1976). Jakob der Lügner. Roman. Frankfurt a. M.: Suhrkamp. (Originalwerk veröffentlicht 1969).

Berg, E., Fuchs, M. (Hrsg.) (1993). Kultur, soziale Praxis. Text. Die Krise der ethnologischen Repräsentation. Frankfurt a.M.: Suhrkamp.

Bermbach, U. (2015). Houston Stewart Chamberlain. Wagners Schwiegersohn - Hitlers Vordenker Stuttgart: Metzler.

\footnotetext{
${ }^{13}$ Hier wäre natürlich an Schillers Spielbegriff zu erinnern, dessen ästhetisch fundierte Gesellschaftstheorie jüngst in postkolonialen, globalen Zusammenhängen wieder aufgenommen wurde (Spivak, 2012).
} 
Blok, A. (1985). Anthropologische Perspektiven. Stuttgart: Klett-Cotta.

Boerner, P. (1975). Das Bild vom anderen Land als Gegenstand literarischer Forschung. Sprache im technischen Zeitalter, 56, S. 313-321.

Butler, J. (1991). Das Unbehagen der Geschlechter. Frankfurt a.M.: Suhrkamp (Originalwerk 1990).

Cappai, G., Shimada, S., \& Straub, J. (Hrsg.) (2010). Interpretative Sozialforschung und Kulturanalyse. Hermeneutik und die komparative Analyse kulturellen Handelns. Bielefeld: transcript.

Cavalli-Sforza, L. \& F. (1994). Verschieden und doch gleich. Ein Genetiker entzieht dem Rassismus die Grundlage. München: Droemer Knaur.

Clausen, D. (1994). Was heißt Rassismus? Darmstadt: WBG.

Corbineau-Hoffmann, A. (2000). Einführung in die Komparatistik. Berlin: E. Schmidt.

Craig, G. A. (1982). Über die Deutschen. München: dtv.

Dreitzel, H. P. (1962). Selbstbild und Gesellschaftsbild: Wissenssoziologische Überlegungen zum Image-Begriff. European Journal of Sociology / Archives Européennes de Sociologie / Europäisches Archiv für Soziologie, 3, No. 2, S. 181-228.

Dyserinck, H. (1981). Komparatistik. Eine Einführung (2. Aufl.). Bonn: Bouvier Grundmann.

Eder, K. (1999). Integration durch Kultur? Das Paradox der Suche nach einer europäischen Identität. In R. Viehoff \& R. T. Segers (Hrsg.), Kultur. Identität. Europa. Über die Schwierigkeiten und Möglichkeiten einer Konstruktion (S. 147-179). Frankfurt a.M.: Suhrkamp.

Elias, N. (1989). Studien über die Deutschen. Machtkämpfe und Habitusentwicklung im 19. und 20. Jahrhundert. Frankfurt a.M.: Suhrkamp.

Foer, J. S. (2003). Alles ist erleuchtet. Roman. Köln: Kiepenheure \& Witsch.

Frank, M. (1986). Die Unhintergehbarkeit von Individualität. Reflexionen über Subjekt, Person und Individuum aus Anlaß ihrer ,postmodernen 'Toterklärung. Frankfurt a.M.: Suhrkamp.

Friedländer, S. (1984). Kitsch und Tod. Der Widerschein des Nazismus. München: Hanser.

Giddens, A. (1995). Konsequenzen der Moderne. Frankfurt a.M.: Suhrkamp.

Goffman, I. (1977). Rahmen-Analyse. Ein Versuch über die Organisation von Alltagserfahrungen. Frankfurt a.M.: Suhrkamp.

Graus, F. (1975). Lebendige Vergangenheit. Überlieferung im Mittelalter und in den Vorstellungen vom Mittelalter. Köln: Böhlau.

Greenblatt, S. (1994). Wunderbare Besitztümer. Die Erfindung des Fremden: Reisende und Entdecker. Berlin: Wagenbach.

Greiffenhagen, M. \& S. (1981). Ein schwieriges Vaterland. Zur politischen Kultur Deutschlands. Frankfurt a.M.: Fischer.

Grözinger, E. (2003). Die schöne Jüdin. Klischees, Mythen und Vorurteile über Juden in der Literatur. Berlin: Philo.

Günther, J. (17. September 2016). Kulturelle Identität - als Problem. Deutsch sein und bleiben. Neue Zürcher Zeitung. Abgerufen von http://www.nzz.ch/feuilleton/aktuell/kulturelle-identitaet-als-prob lem-deutsch-sein-und-bleiben-ld.117226

Guyard, M.-F. (1951). La Littérature Comparée. Paris: PUF.

Hahn, H. H. \& Traba, R. (Hrsg.) (2012). Deutsch-Polnische Erinnerungsorte. Bd. 3: Parallelen. Paderborn u.a.: Schöningh.

Hartman, G. (1996). Das beredte Schweigen der Literatur. Frankfurt a.M.: Suhrkamp.

Hegel, G. W. F. (1970). Grundlinien der Philosophie des Rechts oder Naturrecht und Staatswissenschaft im Grundrisse. Frankfurt a. M.: Suhrkamp (Originalwerk veröffentlicht 1821).

Herder, J. G. (2002). Journal meiner Reise im Jahr 1769 (Historisch-kritische Ausgabe hrsg. von K. Mommsen). Stuttgart: Reclam.

Herodot (1991). Historien (W. Marg Übers.). 1. Bd. Buch I-V. München dtv.

Hertz, F. O. (1904). Moderne Rassentheorien. Kritische Essays. Wien: Stern. 
Hertz, F. (1931). Rasse. In A. Vierkandt (Hrsg.), Handwörterbuch der Soziologie. Stuttgart: Enke, S. 458-466.

Hofmann, M. (2006). Interkulturelle Literaturwissenschaft. Eine Einführung. Paderborn: Fink.

Hofstede, G. (1991). Cultures and Organizations. Software of the Mind. London: McGraw-Hill.

Hortmann, W. (2001). Shakespeare und das deutsche Theater des XX. Jahrhunderts. Mit einem Kapitel über Shakespeare auf den Bühnen der DDR von Maik Hamburger. Berlin: Henschel.

Johnston, O. W. (1990). Der deutsche Nationalmythos. Ursprung eines politischen Programms. Stuttgart: Metzler.

Keupp, H. (1999). Identitätskonstruktionen. Das Patchwork der Identitäten in der Spätmoderne. Reinbek bei Hamburg: Rowohlt.

Kiefer, B. \& Nell, W. (2005). Tradition und Aktualität der Komparatistik im Zeitalter der Medien. In dies. (Hrsg.), Das Gedächtnis der Schrift. Perspektiven der Komparatistik, Wiesbaden: Deutscher Universitätsverlag, S. 1-6.

Klausnitzer, R. (1999). Blaue Blume unterm Hakenkreuz. Die Rezeption der deutschen literarischen Romantik im Dritten Reich. Paderborn: Schöningh.

Kluge, A. (1979). Die Patriotin. Texte/Bilder 1-6. Frankfurt a.M.: Zweitausendeins.

Knapp, R. (2005). Gebrauchsanweisung für Polen. München Zürich: Piper.

Kobylińska, E., Lawaty, A. \& Stephan, R. (Hrsg.). (1992). Deutsche und Polen. 100 Schlüsselbegriffe. München Zürich: Piper.

Koebner, T. \& Pickerodt, G. (Hrsg.). (1987). Die andere Welt. Studien zum Exotismus. Frankfurt a.M.: Athenäum.

Kohl, K.-H. (Hrsg.). (1982). Berliner Festspiele: Mythen der Neuen Welt. Berlin: Frölich \& Kaufmann.

Krockow, C. Graf von (1983). Gewalt für den Frieden? Die politische Kultur des Konflikts. München Zürich: Piper.

Krockow, C. Graf von (1995). Von deutschen Mythen. Rückblick und Ausblick. Stuttgart: DVA.

Krockow, C. Graf von (1999). Über die Deutschen. München: List.

Lawaty, A., Orłowski, H. (Hrsg.). (2003). Deutsche und Polen. Geschichte Kultur Politik. München: C.H. Beck.

Mann, G. (Hrsg.). (1973). Biologismus im 19. Jahrhundert. Stuttgart: Enke.

Marquard, O. (2000). Narrare necesse est. In ders.: Philosophie des Stattdessen. Studien. Stuttgart: Reclam, S. 60-65.

Mayer, A. J. (1984). Adelsmacht und Bürgertum. Die Krise der europäischen Gesellschaft 1848-1914. München: Beck.

Möller, S. (2008). Viva Polonia. Als deutscher Gastarbeiter in Polen. Frankfurt a. M.: Scherz.

Montaigne, M. de (1972). De la vanité. In ders. Essais (Édition présentée, établié et annotée par Pierre Michel). Tome III. Paris: Livre de Poche, S. 201-271 (Originalwerk 1580).

Mosse, G. L. (1990). Die Geschichte des Rassismus in Europa. Frankfurt a.M.: Fischer

Münkler, H., Storch, W. (1988). Siegfrieden. Politik mit einem deutschen Mythos. Berlin: Rotbuch.

Münkler, H. (17. September 2015). Verführer der Deutschen. Udo Bermbachs Studie über Houston Stewart Chamberlain - den Mann, der Hitler den Weg bereitete. Die Zeit, 38. Abgerufen von http://www.zeit.de/2015/38/herfried-muenkler-houston-stewart-chamberlain

Nashe, T. (1985). The Unfortunate Traveller and other Works. Ed. By J.B. Staine. Harmondsworth: Penguin Classics.

Nell, W. (2001). Reflexionen und Konstruktionen des Fremden in der europäischen Literatur. Literarische und sozialwissenschaftliche Studien zu einer interkulturellen Hermeneutik. St. Augustin: Gardez! Verlag.

Nell, W. (2013). Friedrich Otto Herz. In: F. Stengel (Hrsg.), Ausgeschlossen. Zum Gedenken an die 1933-1945 entlassenen Hochschullehrer der Martin-Luther-Universität Halle-Wittenberg (S. 169-182). Halle: Universitätsverlag. 
Nell, W. (2016a). „Ich verstand weder Pilot noch Wirthin...“ Bericht von der Produktivität und den Ambivalenzen eine Kulturschocks. Herders ,Journal meiner Reise im Jahr 1769`. In M-T. Mäder, Ch. Metzger, S. Neubert, A. P. Oloukpona-Yinnon \& L. Schellenberg (Hrsg.), Brücken bauen. Kulturwissenschaft aus interkultureller und multidisziplinärer Perspektive. Festschrift für Dorothee Röseberg zum 65. Geburtstag (S. 81-95). Bielefeld: transcript.

Nell, W. (2016b). Die ,Ideen von 1914‘. Deutsch-französische Perspektiven hundert Jahre später. In A. Richter u.a. (Hrsg.). Der 1. Weltkrieg. Historiographie, Literatur, Medien. Gedächtnispolitik. Berlin (im Druck).

Orłowski, H. (2005). Die Lesbarkeit von Stereotypen. Der deutsche Polendiskurs im Blick historischer Stereotypenforschung und historischer Semantik. Wrocław Görlitz: Neisse.

Orłowski, H. \& Karolak, C. (2015). Pejzaż kulturowy zjednoczonych Niemiec. In C. Karolak, W. Kunicki \& H. Orłowski. Dzieje kultury niemieckiej (S. 538-569). Warszawa: Wydawnictwo Naukowe PWN.

Osgood, C., Suci, G. J. \& Tannenbaum, P. H. (1957). The Measurement of Meaning. Urbana: Univ. of Illinois Press.

Ostasien-Institut Ludwigshafen. (o. J.) Hunnenrede. Ostasienlexikon. Abgerufen am 19. Oktober 2016 von http://www.oai.de/de/publikationen/ostasienlexikon/h/53-ostasienlexikon/hhh/1415-hunnenrede.html

Pasternack, P. \& Sackmann, R. (Hrsg.). (2013). Vier Anläufe: Soziologie an der Universität HalleWittenberg. Bausteine zur lokalen Biographie des Fachs vom Ende des 19. bis zum Beginn des 21. Jahrhunderts. Halle: Mitteldeutscher Verlag.

Pochat, G. (1997). Das Fremde im Mittelalter. Darstellung in Kunst und Literatur. Würzburg: Echter.

Pollak, M. (1990). Rassenwahn und Wissenschaft. Frankfurt a.M.: Hain.

Pross, H. (Hrsg.) (1983). Die Zerstörung der deutschen Politik. Dokumente 1871-1933. Frankfurt a.M.: Fischer.

Raynal, G. \& Diderot, D. (1988). Die Geschichte der beiden Indien (Ausgewählt und erläutert von H.-J. Lüsebrink). Nördlingen: Greno (Originalwerk veröffentlicht 1789).

Renan, E. (1993). Was ist eine Nation? Vortrag in der Sorbonne am 11. März 1882 (Original 1882). In M. Jeismann \& H. Ritter (Hrsg.), Grenzfälle. Über neuen und alten Nationalismus (S. 290-311). Leipzig: Reclam.

Rösgen, P. (Hrsg.). Krauts-Fritz-Piefkes ...? Deutschland von außen. Begleitbuch zur Ausstellung im Haus der Geschichte der Bundesrepublik Deutschland, Bonn, 19. November 1999 bis 26. März 2000. Bonn: Bouvier Verlag 1999.

Saunders, D. (3. November 2015). Two untold histories of Canadian immigration. The Walrus talks Cities of Migration. Toronto. 28. Oktober 2015 [Videodatei]. Abgerufen von https://www.youtube. $\mathrm{com} /$ watch? $\mathrm{v}=\mathrm{j} 74 \mathrm{mRMu} 94 \mathrm{Jo}$

Schulze, H. (1989). Gibt es überhaupt eine deutsche Geschichte? Berlin: Siedler.

Schwarz, E. (1976). Die sechste Schwierigkeit beim Schreiben der Wahrheit. Zum Gruppendenken in Literatur und Leben. In W. Paulsen (Hrsg.), Die USA und Deutschland. Wechselseitige Spiegelungen in der Literatur der Gegenwart. Zum zweihundertjährigen Bestehen der USA am 4. Juli 1976 (S. 11-26). Bern München: Francke 1976.

Seib, W. \& Koch, R. (1989). 1914-1918. Feindbilder [Katalog der Frankfurter Ausstellung]. Frankfurt a.M.: Saalbau.

Simmel, G. (1989). Bemerkungen zu socialethischen Problemen. In Ders. Aufsätze 1887 bis 1890. Über sociale Differenzierung. Die Probleme der Geschichtsphilosophie (1892) (Hrsg. von H.-J. Dahme) (S. 20-36). Frankfurt a.M.: Suhrkamp (Original 1888).

Spiegel. (22. Juli 1964). Der häßliche Deutsche [Titelseite]. Der Spiegel, Nr. 30. Abgerufen von https://www. spiegel-antiquariat.de/der-spiegel-1960-1969/der-spiegel-1964/juli/1540/der-spiegel-nr.-30-br-/22.7. 1964-bis-28.7.1964 
Spivak, G. C. (2012). An Aesthetic Education in the Era of Globalization. Cambridge/Mass.: Harvard UP. Stanzel, F. K. (1998). Europäer. Ein imagologischer Essay. Heidelberg: Winter.

Stierstorfer, K. (Hrsg.). (2003). Deutschlandbilder. Im Spiegel anderer Nationen: Literatur, Presse, Film, Funk, Fernsehen. Reinbek bei Hamburg: Rowohlt.

Szczypiorski, A. (1988). Die schöne Frau Seidenmann. Zürich: Diogenes.

Tacitus (1971). Germania (Übers., erläutert und Nachwort von M. Fuhrmann). Stuttgart: Reclam.

Thomas, A. (2005). Grundlagen der interkulturellen Psychologie. Nordhausen: Bautz.

Waldenfels, B. (2006). Grundmotive einer Phänomenologie des Fremden. Frankfurt a. M.: Suhrkamp.

Weber, E. (1976). Peasants into Frenchmen. The modernization of rural France 1870-1914. Stanford: UP.

Wiegels, R. \& Woesler, W. (Hrsg.) (2003). Arminius und die Varusschlacht. Geschichte - Mythos Literatur (3. Aufl.). Paderborn: Schöningh.

Wikipedia (2016). Völkertafel [Stichwort]. Wikipedia.de. Abgerufen von https://de.wikipedia.org/wiki/ V\%C3\%B6lkertafel_(Steiermark)

Wolfram, G. (2005). Samuels Reise. Stuttgart: DVA.

Zieliński, Z. (Hrsg.) (1995). Polacy Niemcy. Przeszłość. Teraźniejszość. Przyszłość. Katowice: Unia.

Zima, P. V. (2003). Aufgaben und Ziele komparatistischer Forschung: Kulturelle Bedingtheit und kulturelle Vielfalt. In A. Wierlacher \& A. Bogner (Hrsg.), Handbuch Interkulturelle Germanistik S. 562-269). Stuttgart: Metzler. 